\title{
Feasibility of a community-based Functional Power Training program for older adults
}

This article was published in the following Dove Press journal:

Clinical Interventions in Aging

\author{
Queenie Lin Ling Tan' \\ Lilian Min Yen Chye' \\ Daniella Hui Min Ng' \\ Mei Sian Chong' \\ Tze Pin $\mathrm{Ng}^{1,2}$ \\ Shiou Liang Wee ${ }^{1,3}$
}

'Frailty Research Program, Geriatric Education and Research Institute (GERI), Singapore; ${ }^{2}$ Department of Psychological Medicine, National University of Singapore, Singapore; ${ }^{3}$ Faculty of Health and Social Sciences, Singapore Institute of Technology, Singapore
Correspondence: Shiou Liang Wee Geriatric Education and Research Institute, 2 Yishun Central 2, Tower E Level 4 GERI Admin, 768024, Singapore Tel +65 680780 I I

Email weeshiouliang@gmail.com
Purpose: Community-based programs can increase and sustain physical activity participation in older adults, even for those who are physically frail. We studied the feasibility and potential effect of a 12-week structured Functional Power Training (FPT) program involving high velocities and low loads for older adults conducted in a common area of their housing estate.

Patients and methods: The structured FPT program was conducted in collaboration with a health promotion social enterprise and a community service provider based in a public housing site. We recruited nine inactive residents as participants to the single, group-based, twice-weekly program. Attendance and adverse event(s) were recorded throughout the program. The Short Physical Performance Battery, Timed Up and Go (TUG), and 30s Sit-to-Stand tests were used to assess functional outcomes pre- and postprogram. The FRAIL Scale was used to assess their frailty status, and a postprogram experience survey was conducted.

Results: Eight subjects (aged $74 \pm 10$ years) completed the program with an average overall attendance of $90.3 \%$, with at least five participants present for each session. Changes in functional outcomes showed a moderate-to-large effect with significant improvement in TUG $(p<0.01)$. In addition, participants either reversed or maintained their frailty status $(p<0.01)$. Overall, the program was perceived to be well structured, engaging, as well as providing physical and psychosocial benefits. No exercise-related adverse events occurred during the program, and participants were keen to recommend this program to others.

Conclusion: Community-based structured FPT is safe and feasible for frail older adults, with the potential to improve function and reverse frailty status.

Keywords: multicomponent exercise, frail older adults, functional performance, communitybased program

\section{Introduction}

Functional decline leading to dependency is a fear that individuals encounter when considering old age. ${ }^{1}$ Mobility impairment in older adults is strongly associated with muscle weakness and low muscle mass, the hallmarks of physical frailty and sarcopenia. ${ }^{2}$ Nevertheless, current consensus proposes that regardless of age, the neuromuscular system has the ability to adapt to appropriate exercise stimulus. ${ }^{3,4}$

Physical activity guidelines for adults aged 65 years and older emphasize engaging in regular resistance training to improve strength and function, manage frailty, and reduce dependency. ${ }^{5,6}$ While resistance training engages low-velocity contractions at $50 \%-80 \%$ of maximal strength, power training is characterized by performing the concentric phase at high velocity (ie, as fast as possible) with a slow eccentric phase to achieve the greatest benefit of muscular power and strength. ${ }^{7}$ Power training is effective and has emerged as an alternative modality to resistance training ${ }^{8}$ to preserve activities of daily living that require quick, forceful motions,,${ }^{4,7}$ even in the most vulnerable group 
of institutionalized adults aged 85 years and more. ${ }^{9}$ Power producing capabilities compared to muscle strength in older adults are strongly associated with performance of daily tasks, such as getting up from a chair, avoiding oncoming traffic, climbing stairs, and the ability to recover from a loss of balance. ${ }^{4,5,7,8,10}$ Furthermore, muscular power deteriorates earlier and faster than muscular strength with age. ${ }^{11}$ Hence, greater emphasis should be placed on improving muscular power in older adults. ${ }^{4,7}$

Despite the widespread benefits of regular exercise, many older adults face multiple barriers such as mobility impairment, poor health, fear of falling, lack of social interaction and support, lack of interest or enjoyment, and bad weather. ${ }^{12,13}$ Although the aforementioned studies have demonstrated that power training can ameliorate muscle weakness, such interventions often involve the use of specialist equipment, and may not be practical for implementation in community settings like housing sites and senior activity centers. ${ }^{14}$ Furthermore, information on exercise programs that place emphasis on high-velocity movements without the use of specialist equipment that can be adopted by community-dwelling older adults is scarce.

In this study, we examined the feasibility and effects of conducting a 12-week structured Functional Power Training (FPT) program within a housing estate. We hypothesized that a community-based structured FPT program involving high velocities and low loads using body weight and simple equipment would be feasible and safe for older adults.

\section{Materials and methods}

A structured 12-week FPT program was conducted with a health promotion social enterprise (ProAge Pte Ltd, Singapore; http://www.proage.sg) and a community service provider (Filos Community Services, Singapore; http://www. filos.sg) based in a public housing site (Chai Chee Estate in the East Singapore). Filos referred suitable participants living within the housing site. Eligibility criteria were as follows: Adults aged 55 years and above, able to ambulate without personal assistance, and able to comprehend simple instructions. We excluded participants who had uncontrolled medical conditions, diagnosed with major depression, severe audiovisual impairment, progressive degenerative neurologic disease, or terminal illness with life expectancy $<12$ months. To ensure exercise safety, we administered the Exercise and Sports Science Australia (ESSA) screening tool to screen for any contraindication to exercise. Subjects with positive response to any question(s) in the ESSA screening tool were referred to a doctor for medical clearance prior to participation. All participants gave written informed consent to the study, which was approved by the National Health Group ethics board.

\section{Tests and measures}

Baseline demographics and health status were obtained using a lifestyle questionnaire, focusing on comorbidity, medications, falls history, and physical activity levels. Anthropometric measures of height and weight were obtained at baseline and postintervention. A series of functional performance measures included the Short Physical Performance Battery (SPPB), Timed Up and Go (TUG), and 30s Sit to Stand (30s STS). A FRAIL Scale questionnaire was administered to categorize participants into frail, prefrail, and robust categories. ${ }^{15}$ At the end of the 12-week FPT program, participants repeated the physical measures. In addition, semistructured interviews were conducted upon study completion to understand participants' experiences and adherence factors. Trained researchers from the Geriatric Education and Research Institute administered the test procedures and interviews.

\section{Program conduct, feasibility, and safety}

Prior to each exercise session, pulse oximetry (ChoiceMMed OxyWatch MD300C63) was used to measure blood oxygen levels and heart rate. Participants with blood oxygen levels below $95 \% \mathrm{SPO}_{2}$ were advised against participation in the session. Other pre-exercise screening criteria included high resting heart rate, ${ }^{16}$ abnormal resting blood pressure, ${ }^{17}$ giddiness, or any form of discomfort. Attendance was recorded for each session. Program adherence was determined by the number of exercise sessions attended against the total number of 24 sessions. We considered the program to be feasible if attendance was maintained at $>50 \%$ for all sessions and averaged $>80 \%$ per session. We monitored and recorded adverse events throughout the course of the program.

\section{Program acceptance, engagement, and adherence}

A survey was conducted after the physical posttest measurements were taken to understand participants' experiences and factors for adherence to the program. The survey comprised nine questions on program acceptance, satisfaction, adherence, engagement, and desire for continued participation, with responses recorded by a four-point Likert scale $(1=$ strongly disagree, $2=$ disagree, $3=$ agree, and $4=$ strongly agree).

\section{Functional outcomes}

\section{Short Physical Performance Battery}

The SPPB is a widely used functional performance test, and has been validated in community-dwelling older 
populations. ${ }^{18}$ The battery comprises the following: 1) timed Sit to Stand, 2) static balance, and 3) timed $2.44 \mathrm{~m}$ walk. ${ }^{19}$ The timed Sit to Stand test assesses one's ability to complete five successful chair rises. A successful chair rise was constituted by a rise to a full standing position with arms crossed over the chest. The static balance task had three levels of difficulty based on foot positioning: side by side, semitandem, and tandem stance. Participants were required to maintain their feet position for 10 seconds to proceed to the next level. The walk test is a timed walk, where participants covered a distance of $2.44 \mathrm{~m}$ at their usual walking pace. For each component, a score of $0-4$ points was awarded based on individual performance. The maximum total score was 12 points, where a higher score meant better physical function.

\section{Timed Up and Go}

The TUG consisted of transfer tasks to assess gait and balance. ${ }^{20}$ The test required participants to stand up from a chair, walk a distance of $3 \mathrm{~m}$ (marked with a cone) at a comfortable pace, turn, walk back, and sit down. Participants were permitted to use routine walking aids, with no additional physical assistance given. The test was performed twice, with the better of two trials recorded.

\section{0-Second Sit to Stand}

As a measure of lower extremity endurance, participants performed as many chair rises to an upright position as possible in 30 seconds. ${ }^{18}$ The number of successful chair rises was recorded.

\section{Frailty classification}

The FRAIL Scale questionnaire comprised five components of the frailty phenotype based on self-report: fatigue, resistance, ambulation, illness, and loss of weight. ${ }^{15}$ One point was allocated if the participant responded "yes" in a component, with the total summed score ranging from 0 to 5 and classified into three frailty statuses: robust (score $=0$ ), prefrail (score $=1-2$ ), or frail (score $=3-5$ ). The scale has been validated in different populations in different settings, including Hong Kong and Singapore, to identify individuals at risk of adverse health outcomes. ${ }^{21,22}$

\section{Intervention program}

The exercise intervention comprised a 12 -week ( 24 sessions) structured FPT program comprising high-velocity movement, balance, and mobility exercises. The sessions were led by a qualified exercise physiologist from ProAge, and assisted by staffs and volunteers from Filos and/or Geriatric Education and Research Institute, with a trainer-to-participants ratio of at least 1:3. Sessions were held at a public area in the housing estate, twice weekly for 60 minutes with at least one rest day between consecutive sessions.

The high-velocity movement training consisted of upper and lower extremity resistance exercises with focus on the lower body muscle groups (hip abductors, adductors, extensors, knee flexors and extensors, ankle plantarflexors, and dorsiflexors). The balance and mobility exercises targeted lower body muscles through the inclusion of weight shifts and reduction of base of support. In each session, participants performed 5-6 lower body and upper body resistance exercises, supplemented with two balance and mobility exercises. Training intensity progressed according to each participant's capability, starting from two sets of 10 repetitions for resistance exercises and two repetitions of 30 seconds for balance and mobility exercises. Examples of the intervention exercises prescribed are listed in Table 1. The 24 sessions were distributed into four progressive phases: 1) Familiarization, 2) Skill-Up, 3) Training Gains, and 4) Optimizing Gains. In the "Familiarization" phase (week 0-2), participants were taught the basic techniques of the exercises in a seated position and familiarization of the training protocol. Emphasis was placed on safety and confidence in executing the exercises in proper form. In the "Skill-Up" phase (week 3-4), participants performed a variety of bodyweight exercises in a standing position. The "Training Gains" phase (week 5-8) included an increase in exercise intensity with more repetitions and the addition of resistance bands. The final "Optimizing Gains" phase (week 9-12) consisted of exercises that required weight transfers, such as circuit training and standing on unstable surfaces. Each session was accompanied with music to

Table I List of intervention exercises

\begin{tabular}{|c|c|}
\hline \multicolumn{2}{|l|}{ Intervention exercises } \\
\hline $\begin{array}{l}\text { Resistance exercise } \\
\text { (lower extremities) }\end{array}$ & $\begin{array}{l}\text { Balance and } \\
\text { mobility exercises }\end{array}$ \\
\hline I. Toe pointing and flexion & I. Seated side reach \\
\hline 2. Toe raise & 2. Seated fly \\
\hline 3. Calf raise & 3. Side toe taps \\
\hline 4. Leg extension & 4. Static balance \\
\hline 4. Hip flexion & 5. Weight shifts \\
\hline 5. Hip extension & 6. Standing march \\
\hline \multicolumn{2}{|l|}{ 6. Hip abduction } \\
\hline \multicolumn{2}{|l|}{ 7. Hip adduction } \\
\hline \multicolumn{2}{|l|}{ 8. Chair rise } \\
\hline \multicolumn{2}{|c|}{ Resistance exercise (upper extremities) } \\
\hline \multicolumn{2}{|l|}{ I. Arm swing } \\
\hline \multicolumn{2}{|l|}{ 2. Seated reverse fly } \\
\hline \multicolumn{2}{|l|}{ 3. Seated chest press } \\
\hline 4. Triceps extension & \\
\hline
\end{tabular}


enhance adoption and maintenance of the program. To maximize social interaction and engagement, effective strategies such as buddy systems and regular positive feedbacks were also implemented.

\section{Data analysis}

Nonparametric Wilcoxon signed-rank test was used to compare pre-posttest measures. Results from the functional tests and FRAIL Scale were reported as mean and standard deviation (SD), and effect size of each test was calculated using Cohen's $d$. Results of the study closure interview was reported as the percentage of responses for each question. The breakdown of the FRAIL Scale was reported as the percentage of participants who responded "Yes" to each component.

\section{Results}

Nine participants were enrolled into the program, and the baseline demographics are described in Table 2. The mean age of the participants was $73 \pm 10$ years. All participants were Chinese and had an average of four chronic illnesses, with hypertension, high cholesterol level, visual impairment, arthritis, and diabetes as the top five common comorbidities. More than $50 \%$ of the participants relied on some form of mobility aids (canes and motorized wheelchair). According

Table 2 Participant demographics ( $\mathrm{N}=9)$

\begin{tabular}{ll}
\hline Demographics & $\begin{array}{l}\text { Mean (SD) or } \\
\text { frequency (\%) }\end{array}$ \\
\hline Females & $77.8 \%$ \\
Chinese & $100 \%$ \\
Age & $73.4(10.0)$ \\
BMI (kg/m ${ }^{2}$ ) & $25.0(3.7)$ \\
Comorbidities & $3.5(1.7)$ \\
Hypertension & $88.9 \%$ \\
High cholesterol level & $77.8 \%$ \\
Visual impairment & $55.6 \%$ \\
Arthritis & $44.4 \%$ \\
Diabetes & $22.2 \%$ \\
Smoking status & \\
Never smoked & $77.8 \%$ \\
Smoker & $22.2 \%$ \\
Alcohol intake & $22.2 \%$ \\
Mobility aids & \\
Independent & $44.4 \%$ \\
Canes & $44.4 \%$ \\
Motorized wheelchair & $11.1 \%$ \\
Physical frailty (FRAlL Scale) & \\
Robust & $11.1 \%$ \\
Prefrail & $44.4 \%$ \\
Frail & $44.4 \%$ \\
\hline Aistory of falls (past 6 months) & $33.3 \%$ \\
\hline
\end{tabular}

Abbreviations: SD, standard deviation; BMI, body mass index. to the FRAIL Scale, four participants were categorized as frail, three as prefrail, and one as robust at baseline.

\section{Feasibility and safety}

The program excluded participants who had uncontrolled medical conditions, or without doctor's medical clearance if they failed the ESSA screening tool. Eight participants completed the 12-week intervention with one dropout after the sixth session due to personal reasons. Attendance for every session is reported in Figure 1, with a minimal attendance of $62.5 \%$ in sessions 7 and 9 . The mean attendance rate for the eight completers was $90.3 \%$. Two participants achieved $100 \%$ attendance for the program. The participant with the lowest attendance rate of $75 \%$ was unable to attend some sessions due to conflict with medical appointments (a 2-week break for gastric evaluation). Two participants took 1-week breaks for personal reasons or minor medical reasons. There were no adverse events related to the exercises during the program.

\section{Program acceptance and satisfactory}

Participants perceived the program to be engaging, with relevant physical, psychological, and social benefits, which have likely contributed to adherence (Table 3 ). The participants also expressed that they would recommend the program to others.

\section{Functional outcomes}

Participants showed improvements in all the functional or FRAIL measures with moderate effects of 0.57 and 0.55 for SPPB score and 30s STS time, respectively, and large effects of 0.86 and 1.23 for TUG and FRAIL Scale scores, respectively (Table 4). There were pre-postprogram reductions in TUG time (13.9 \pm 2.5 to $11.8 \pm 2.3$ seconds; $p=0.01)$ and FRAIL score $(2.0 \pm 1.2$ to $0.9 \pm 0.6 ; p=0.01)$.

All frail participants reversed their frail score to the prefrail category (Table 4). Three participants who were categorized as prefrail had their frailty score improved with one participant who reversed into the robust category. The breakdown of the five components of the FRAIL Scale in Figure 2 reflects the corresponding improvements in response to fatigue and mobility components of FRAIL, which were more pronounced, followed by endurance and weight loss than illnesses.

\section{Discussion}

Frailty is not a contraindication to exercise, but an important reason to prescribe it. ${ }^{6}$ Community-based programs are designed to reach older people in "real-world" settings 


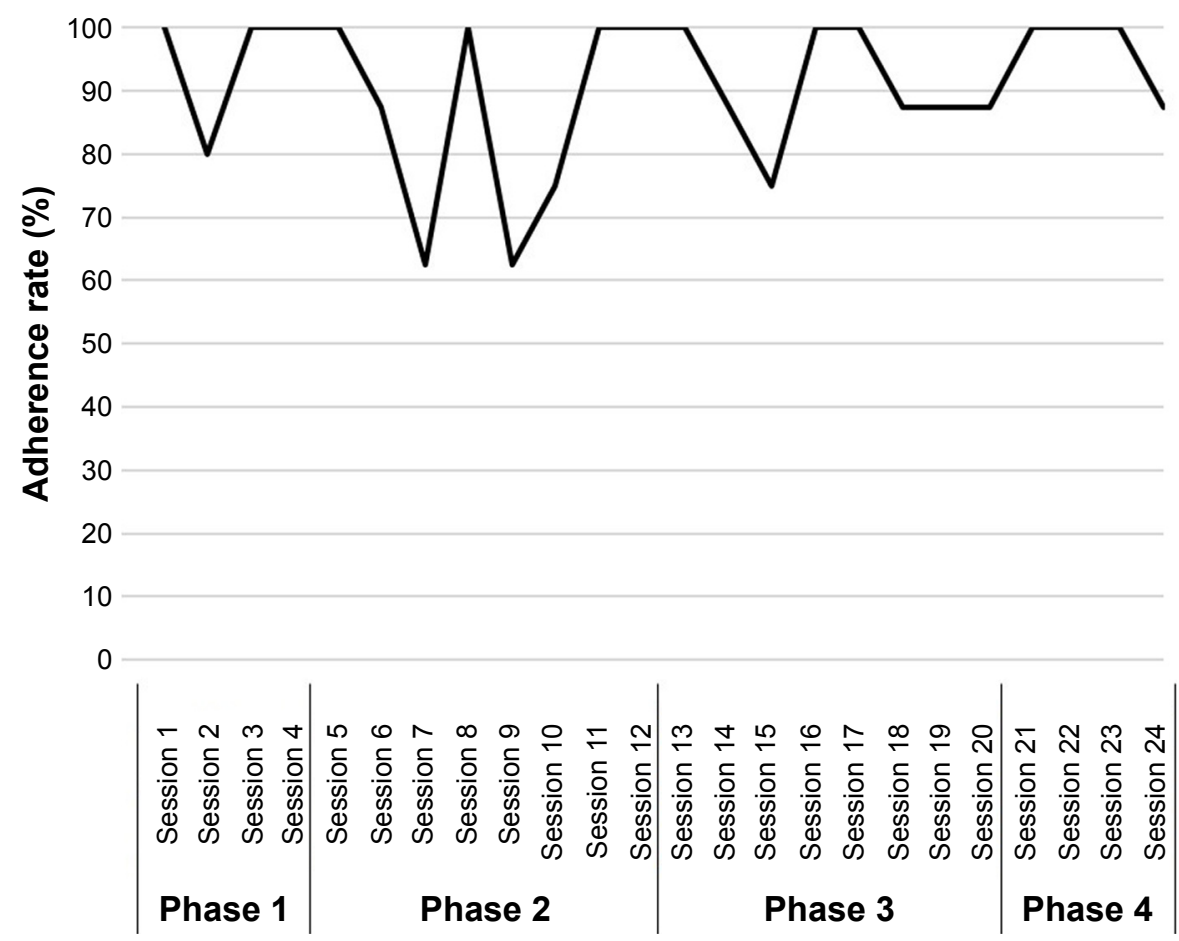

Figure I Adherence rate for each session of the program.

outside the traditional health care approach. We found that a structured FPT program for frail residents based in the public housing site is feasible and safe.

Frail persons are at risk of disability, falls, hospitalization, and use of nursing homes; early intervention with frail persons will improve quality of life and reduce care

Table 3 Responses from feedback questionnaires

\section{Responses ( $\mathbf{N}=\mathbf{8})$}

I. The exercise program was well structured, organized, and easy to follow.

$$
\text { SD }=0 \quad \mathrm{D}=0 \quad \mathrm{~A}=5 \quad \mathrm{SA}=3
$$

2. The exercise program was fun, enjoyable and engaging.

$$
\mathrm{SD}=0 \quad \mathrm{D}=0 \quad \mathrm{~A}=5 \quad \mathrm{SA}=3
$$

3. The exercise program was relevant to my daily activities.
$\mathrm{SD}=0$
$\mathrm{D}=0$
$A=4$
$\mathrm{SA}=4$

4. The exercise program helped to improve my social interaction with other participants.

$\mathrm{SD}=0 \quad \mathrm{D}=0 \quad \mathrm{~A}=4 \quad \mathrm{SA}=4$

5. After the exercise program, I feel more energetic and happy.
$\mathrm{SD}=0$
$\mathrm{D}=0$
$\mathrm{A}=7$
$S A=I$

6. After the exercise program, I feel stronger and confident with daily activities.

$\mathrm{SD}=0 \quad \mathrm{D}=0 \quad \mathrm{~A}=5 \quad \mathrm{SA}=3$

7. I feel that I have benefited from the exercise program.

$\mathrm{SD}=0 \quad \mathrm{D}=0 \quad \mathrm{~A}=4 \quad \mathrm{SA}=4$

8. I will recommend this exercise program to others.

$$
\mathrm{SD}=0 \quad \mathrm{D}=0 \quad \mathrm{~A}=6 \quad \mathrm{SA}=2
$$

9. I will participate in such exercise program in the future.
$\mathrm{SD}=0$
$\mathrm{D}=\mathrm{I}$
$\mathrm{A}=4$
$\mathrm{SA}=3$

Abbreviations: $S D$, strongly disagree; $D$, disagree; $A$, agree; $S A$, strongly agree. burden. ${ }^{23,24}$ The strong adherence in the program resulted in significant improvement in physical function. Importantly, the structured program also showed potential to reverse frailty in all four frail and one prefrail participants. The improvement in functional outcomes as measured by TUG $(18 \%)$ and SPPB (14\%) is comparable to the findings of a recent review on power training for older persons. ${ }^{9}$ Although most of the participants were physically frail or prefrail, program adherence was high with no report of exercise-related adverse event. This suggests that structured FPT can be a prescribed modality of exercise for older adults in replacement of traditional resistance exercise. As no specialized equipment is required, these exercises can also be practiced at home over and above the group-based program.

Research in physical training in frail and/or older adults is dominated by short-term interventions, producing short-term gains with little consideration of the long-term maintenance of functional performance. ${ }^{9}$ The proportion of people who do not meet the physical activity guidelines are higher after they attain the age of 60 years, with marked increases after age 80 years, where nearly half the populations did not meet the minimal threshold for health. ${ }^{6}$ With Singapore's aging population, it is important to have effective and sustainable community-based programs to support the long-term maintenance of physical function of older people. Attendance for this community-based program was at least $62.5 \%$ for any session, with eight of nine participants completing the 
Table 4 Physical performances during baseline and postintervention

\begin{tabular}{|c|c|c|c|c|c|c|c|c|c|c|}
\hline \multirow[t]{2}{*}{ Measures } & \multirow[t]{2}{*}{ Mean \pm SD } & \multicolumn{9}{|c|}{ Participant } \\
\hline & & $\mathbf{I}$ & 2 & 3 & 4 & 5 & 6 & 7 & 8 & $d$ \\
\hline \multicolumn{11}{|l|}{ SPPB score (0-12 pts) } \\
\hline Baseline & $8.4 \pm 1.9^{a}$ & 9 & 10 & 6 & 9 & 10 & 8 & 10 & 5 & 0.57 \\
\hline Timed Sit to Stand & $2.5 \pm 1.2$ & 3 & 4 & 4 & 4 & 3 & I & 4 & 2 & \\
\hline Static balance & $2.7 \pm 1.2$ & 4 & 4 & 2 & I & 4 & 2 & 3 & 2 & \\
\hline Timed $2.44 \mathrm{~m}$ walk & $3.1 \pm 0.6$ & 3 & 3 & 3 & 3 & 3 & 3 & 3 & 3 & \\
\hline Post & $9.6 \pm 2.4$ & 10 & 12 & 11 & 12 & 10 & 5 & 10 & 7 & \\
\hline Timed Sit to Stand & $3.1 \pm 1.1$ & 3 & 4 & 4 & 4 & 3 & I & 4 & 2 & \\
\hline Static balance & $3.5 \pm 0.8$ & 4 & 4 & 4 & 4 & 4 & 2 & 3 & 3 & \\
\hline Timed $2.44 \mathrm{~m}$ walk & $3.0 \pm 0.8$ & 3 & 4 & 3 & 4 & 3 & 2 & 3 & 2 & \\
\hline \multicolumn{11}{|l|}{ TUG $(s)^{\mathrm{a}}$} \\
\hline Baseline & $13.9 \pm 2.5$ & 12.4 & 14.7 & 15.5 & 10.4 & 11.7 & 16.8 & 12.3 & 16.9 & 0.86 \\
\hline Post & $11.8 \pm 2.3$ & 11.9 & 9.5 & 13.4 & 8.2 & 11.0 & 14.7 & 11.0 & 14.6 & \\
\hline \multicolumn{11}{|l|}{ 30s STS } \\
\hline Baseline & $11.6 \pm 2.9$ & 13 & 15 & 11 & 13 & 10 & 9 & 15 & 7 & 0.55 \\
\hline Post & $13.1 \pm 2.6$ & 13 & 18 & 13 & 13 & 15 & 10 & 13 & 10 & \\
\hline \multicolumn{11}{|l|}{ Frailty score $(0-5)^{a}$} \\
\hline Baseline & $2.0 \pm 1.2$ & 3 & I & 3 & 3 & I & 3 & 0 & 2 & 1.23 \\
\hline Post & $0.9 \pm 0.6$ & 2 & 0 & I & I & I & I & 0 & I & \\
\hline
\end{tabular}

Note: aSignificant difference between baseline and postmeasurements $(p=0.0 \mathrm{I})$.

Abbreviations: $d=$ Cohen's $d$ (calculated as mean/standard deviation [SD]); pt, points; SPPB, Short Physical Performance Battery; TUG, Timed Up and Go; 30s STS, 30s Sit-to-Stand tests.

program. The high mean adherence $(90.3 \%)$ for those who completed the program can be explained by the survey results: that the program was well structured, relevant to daily tasks, engaging, and conferred physical and psychosocial benefits. The finding that all participants would recommend the program to others, and all except one would continue to participate in similar programs, suggests that our structured FPT program has the potential for wider, sustained participation. Compared to conventional resistance training, our FPT program required no specialist equipment for loading and would be feasible for such a small group-based program in a housing site, with convenient and easy accessibility for the

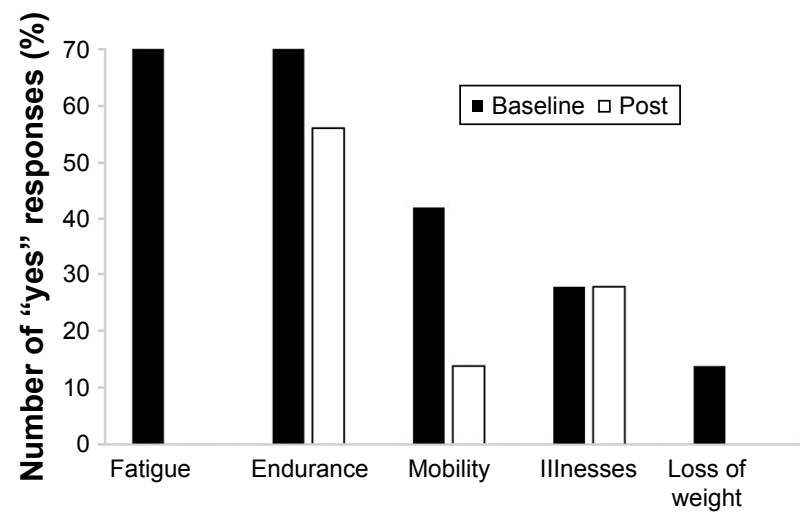

Figure 2 Comparison of baseline and postmeasurement of the individual components of FRAIL Scale. residents. Community partnerships demonstrated in this study would be critical to the sustainability of such a program in a public housing setting. ${ }^{6,12}$ The result of this feasibility study would inform the implementation of a larger-scale community-based multisite, randomized controlled intervention trial being planned to target these frail and prefrail individuals, given their high prevalence in the community. ${ }^{25}$

From the individual components of the FRAIL scale, the frail participants reversed to prefrail as they reported "no" to the mobility question "By yourself, without using any aids, do you have any difficulty walking to a bus stop?" by the end of the exercise program. Additionally, all participants reported that they "do not feel tired most of the time during the past 4 weeks" to the fatigue question that was a major contributing factor to the decreased FRAIL score (Figure 2). This suggests that the structured FPT program resulted in improved mobility and lesser fatigue.

The improvement in TUG suggests that the FPT program has the potential to improve balance. Six out of seven prefrail/ frail participants showed a clinically meaningful change in SPPB score. ${ }^{21,22}$ When comparing the individual components of SPPB, the improvement in SPPB scores was mainly contributed by a faster five times Sit to Stand time and standing balance, rather than gait speed. This suggests that the structured FPT has the potential to improve not only muscle strength but also postural stability. ${ }^{26}$ Lastly, the training 
intensity may not be sufficient to elicit similar improvement in functional outcomes in the robust participant.

\section{Strengths and limitations}

This purpose of this feasibility study is to optimize the structured FPT intervention. The strengths include the implementation in a "real-world" housing site setting where the participants reside, close partnership with local community providers to engage participation and adherence, and using simple equipment such as chairs and resistance bands. However, we felt that the training intensity of the final "Optimizing Gains" phase could be of greater intensity and difficulty. Our results are only applicable to this program and could potentially be different had the intensity been higher. In addition, an objective measure of physical frailty such as the Fried phenotype rather than the FRAIL self-report scale could better quantify the change in frailty status. Furthermore, we did not conduct a follow-up on the participants' fall frequency, an important factor to frailty. Owing to its small sample size, the study was implemented as a feasibility study. Thus, we could not make any assumptions on the effectiveness of the FPT intervention. However, the data collected would provide information to establish further investigation. A larger randomized controlled trial to determine the efficacy of the FPT intervention is currently underway.

\section{Conclusion}

Our study suggests that it is feasible and safe for frail and prefrail community-dwelling older adults to complete a structured 12-week high-volume, moderate-intensity FPT exercise program. Conducting the program in collaboration with a health promotion social enterprise, and working with a site-based community service provider, there is potential for a larger multisite randomized control study to examine the effectiveness of such a program to improve function and reverse frailty.

\section{Acknowledgments}

The authors gratefully acknowledge the partnership and support of ProAge Pte Ltd and Filos Community Services. The authors thank Dr Teo Wei Peng (Deakin University, Australia) for his inputs with regard to the implementation of the program.

\section{Disclosure}

The authors report no conflicts of interest in this work.

\section{References}

1. Lustig C, Shah P, Seidler R, Reuter-Lorenz PA. Aging, training and the brain: a review and future directions. Neuropsychol Rev. 2009; 19(4):504-522.

2. Fiatarone MA, O’Neill EF, Ryan ND, et al. Exercise training and nutritional supplementation for physical frailty in very elderly people. N Engl J Med. 1994;330(25):1769-1775.

3. Landi F, Marzetti E, Martone AM, Bernabei R, Onder G. Exercise as a remedy for sarcopenia. Curr Opin Clin Nutr Metab Care. 2014; 17(1):25-31.

4. Tschopp M, Sattelmayer MK, Hilfiker R. Is power training or conventional resistance training better for function in elder persons? A meta-analysis. Age Aging. 2011;40(5):549-556.

5. Chodzko-Zajko WJ, Proctor DN, Singh MAF, et al. American College of Sports Medicine position stand. Exercise and physical activity for older adults. Med Sci Sports Exerc. 2009;41(7):1510-1530.

6. Bauman A, Merom D, Bull FC, Buchner DM, Fiatarone Singh MA. Updating the evidence for physical activity: summative reviews of the epidemiological evidence, prevalence, and interventions to promote “active aging”. Gerontologist. 2016;56(supp1 2):S268-S280.

7. Porter MM. Power training for older adults. Appl Physiol Nutr Metab. 2006;31(2):87-94.

8. Bryne C, Faure C, Keene DJ, Lamb SE. Aging, muscle power and physical function: a systematic review and implications for pragmatic training interventions. Sports Med. 2016;46(9):1311-1332.

9. Cadore EL, Casas-Herrero A, Zambom-Ferraresi F, et al. Multicomponent exercises including muscle power training enhance muscle mass, power output, and functional outcomes in institutionalized frail nonagenarians. Age. 2014;36(2):773-785.

10. Aagaard P, Simonsen EB, Andersen JL, Magnusson P, Dyhre-Poulsen P. Increased rate of force development and neural drive of human skeletal muscle following resistance training. J Appl Physiol. 2002; 93(4):1318-1326.

11. Reid KF, Fielding RA. Skeletal muscle power: a critical determinant of physical functioning in older adults. Exerc Sport Sci Rev. 2012; 40(1):4-12.

12. Baert V, Gorus E, Mets T, Geerts C, Bautmans I. Motivators and barriers for physical activity in the oldest old: a systematic review. Aging Res Rev. 2011;10(4):464-474.

13. Moschny A, Platen P, Klaaßen-Mielke R, Trampisch U, Hinrichs T. Barriers to physical activity in older adults in Germany: a cross-sectional study. Int J Behav Nutr Phys Act. 2011;8(1):121.

14. Brach JS. Community-based exercise programs for older adults. In: Sullivan GM, Pomidor AK, editors. Exercise for Aging Adults. Champaign, IL: Springer; 2015:131-138.

15. Morley JE, Malmstrom TK, Miller DK. A simple frailty questionnaire (FRAIL) predicts outcomes in middle aged African Americans. J Nutr Health Aging. 2012;16(7):601-608.

16. Umetani K, Singer DH, McCraty R, Atkinson M. Twenty-four hour time domain heart rate variability and heart rate: relations to age and gender over nine decades. J Am Coll Cardiol. 1998;31(3):593-601.

17. Steffen TM, Hacker TA, Mollinger L. Age-and gender-related test performance in community-dwelling elderly people: six-minute walk test, berg balance scale, timed up \& go test, and gait speeds. Phys Ther. 2002;82(2):128-137.

18. Mijnarends DM, Meijers JMM, Halfens RJG, et al. Validity and reliability of tools to measure muscle mass, strength, and physical performance in community-dwelling older people: a systematic review. J Am Med Dir Assoc. 2013;14(3):170-178.

19. Guralnik JM, Simonsick EM, Ferrucci L, et al. A short physical performance battery assessing lower extremity function: association with self-reported disability and prediction of mortality and nursing home admission. J Gerontol. 1994;49(2):M85-M94.

20. Podsiadlo D, Richardson S. The timed "Up \& Go": a test of basic functional mobility for frail elderly persons. J Am Geriatr Soc. 1991;39(2): 142-148. 
21. Woo J, Yu R, Wong M, Yeung F, Wong M, Lum C. Frailty screening in the community using the FRAIL scale. J Am Med Dir Assoc. 2015; 16(5):412-419.

22. Chong E, Ho E, Baldevarona-Llego J, Chan M, Wu L, Tay L. Frailty and risk of adverse outcomes in hospitalized old adults: a comparison of different frailty measures. J Am Med Dir Assoc. 2017;18(7): 638.e7-638.e11.

23. Morley JE, Vellas B, van Kan GA, et al. Frailty consensus: a call to action. J Am Med Dir Assoc. 2013;14(6):392-397.

24. Clegg A, Young J, Iliffe S, Rikkert MO, Rockwood K. Frailty in elderly people. Lancet. 2013;381(9868):752-762.
25. Wei K, Nyunt MSZ, Gao Q, Wee SL, Ng TP. Frailty and malnutrition: related and distinct syndrome prevalence and association among community-dwelling older adults: singapore longitudinal aging studies. J Am Med Dir Assoc. 2017;18(12):1019-1028.

26. Zech A, Drey M, Freiberge E, et al. Residual effects of muscle strength and muscle power training and detraining on physical function in community-dwelling prefrail older adults: a randomized controlled trial. BMC Geriatr. 2012;12(1):68.
Clinical Interventions in Aging

\section{Publish your work in this journal}

Clinical Interventions in Aging is an international, peer-reviewed journal focusing on evidence-based reports on the value or lack thereof of treatments intended to prevent or delay the onset of maladaptive correlates of aging in human beings. This journal is indexed on PubMed Central, MedLine,

\section{Dovepress}

CAS, Scopus and the Elsevier Bibliographic databases. The manuscript management system is completely online and includes a very quick and fair peer-review system, which is all easy to use. Visit http://www.dovepress. com/testimonials.php to read real quotes from published authors. 